\title{
Association Between Bronchiolitis Patient Volume and Continuous Pulse Oximetry Monitoring in 25 Hospitals
}

\author{
Patricia A Stoeck, MD ${ }^{1,2 *}$, Deanna F Chieco, MD, EdM1,2, Elizabeth W Pingree, MD ${ }^{1,2}$, Christopher P Landrigan, MD, MPH ${ }^{1,2,3}$, \\ Christopher P Bonafide, MD, MSCE ${ }^{4,5,6}$, for the Pediatric Research in Inpatient Settings (PRIS) Network
}

\begin{abstract}
${ }^{1}$ Division of General Pediatrics, Department of Pediatrics, Boston Children's Hospital, Boston, Massachusetts; ${ }^{2}$ Harvard Medical School, Boston, Massachusetts; ' ${ }^{3}$ ivision of Sleep and Circadian Disorders, Departments of Medicine and Neurology, Brigham and Women's Hospital, Boston, Massachusetts; ${ }^{4}$ Section of Pediatric Hospital Medicine, Children's Hospital of Philadelphia, Philadelphia, Pennsylvania; ${ }^{5}$ Center for Pediatric Clinical Effectiveness, Children's Hospital of Philadelphia, Philadelphia, Pennsylvania; ${ }^{6}$ Department of Pediatrics, Perelman School of Medicine at the University of Pennsylvania, Philadelphia, Pennsylvania.
\end{abstract}

Continuous pulse oximetry monitoring in stable patients with bronchiolitis is discouraged by national guidelines in order to reduce overuse, yet wide practice variation exists among hospitals. Understanding the association between monitoring overuse and hospital unit-level factors may identify areas for improvement. Conducted at 25 sites from the Pediatric Research in Inpatient Settings (PRIS) Network's Eliminating Monitoring Overuse (EMO) study, this substudy used data from 2,366 in-person observations of pulse oximetry use in patients with bronchiolitis to determine whether hospital unit-level factors were associated with variation in pulse oximetry use for patients in whom continuous monitoring is not indicated. Hospital units were classified by bronchiolitis admission burden. Monitoring rates were analyzed in a mixed-effects model that accounted for variation in baseline monitoring rates among hospitals and adjusted for covariates significantly associated with continuous pulse oximetry monitoring use in the primary study's analysis. Low burden units $(<10 \%$ of total admissions) had a 2.16-fold increased odds of pulse oximetry overuse compared to high burden units ( $\geq 40 \%$ of total admissions) $(95 \% \mathrm{Cl}, 1.27-3.69 ; P=.01)$. These results suggest that units caring for a lower percentage of patients with bronchiolitis are more likely to overuse pulse oximetry despite national guidelines. Journal of Hospital Medicine 2020; 15:669-672. (C) 2020 Society of Hospital Medicine ontinuous pulse oximetry monitoring in children with bronchiolitis who don't require supplemental oxygen is discouraged by practice guidelines and is recognized as a form of medical overuse. ${ }^{1-3}$ This practice can be associated with negative outcomes, including prolonged length of stay, ${ }^{4-6}$ increased cost of hospitalization, ${ }^{7}$ and alarm fatigue among nurses. ${ }^{8}$ Despite initiatives to reduce continuous pulse oximetry monitoring in stable patients with bronchiolitis, ${ }^{1,2}$ wide practice variation exists between hospitals. ${ }^{9,10}$ Previous studies have shown that higher prevalence of inpatient bronchiolitis admissions is associated with decreased utilization of unnecessary interventions. ${ }^{11}$ However, the relationship between pulse oximetry use and bronchiolitis prevalence has not been studied. The objective of this study is to test the hypothesis that hospital units with lower proportions of patients admitted for bronchiolitis and those with fewer general pediatrics patients relative to subspecialty patients would have higher rates of pulse oximetry overuse.

*Corresponding Author: Patricia A Stoeck, MD; Email: patricia.stoeck@ childrens.harvard.edu; Twitter: @pattystoeck.

Published online first October 21, 2020

Find additional supporting information in the online version of this article.

Received: April 16, 2020; Revised: August 6, 2020; Accepted: August 8, 2020

(๔) 2020 Society of Hospital Medicine DOI 10.12788/jhm.3520

\section{METHODS}

\section{Study Design}

We conducted a substudy of the Pediatric Research in Inpatient Settings (PRIS) Network's Eliminating Monitoring Overuse (EMO) pulse oximetry study, ${ }^{10,12}$ a 56-hospital cross-sectional study that used direct observation to measure the prevalence of continuous pulse oximetry monitoring in hospitalized infants with bronchiolitis who did not require supplemental oxygen between December 1, 2018, through March 31, 2019. This substudy was not included as part of the original aims of the project and was proposed as a separate analysis during data collection. For US sites, the Institutional Review Board (IRB) at Children's Hospital of Philadelphia approved the study and served as the central IRB. The Research Ethics Board at University of Calgary also approved the study.

\section{Site Selection}

Hospitals with at least 60 observations were eligible for inclusion. Of the 32 hospitals that conducted the minimum observations, 25 agreed to participate ( 21 free-standing children's hospitals, 3 children's hospitals within general hospitals, and 1 community hospital).

\section{Patient Population}

The parent study included patients aged 8 weeks through 23 months with a primary diagnosis of bronchiolitis. Patients were 
TABLE. Pulse Oximetry Monitoring by Bronchiolitis Admission Burden Category

\begin{tabular}{|c|c|c|c|}
\hline Unit's bronchiolitis admission burden ${ }^{a}$ & $\begin{array}{l}\text { No. bronchiolitis bedside observations } \\
\qquad(\mathrm{N}=2,366)\end{array}$ & $\begin{array}{l}\text { Unadjusted observed use, } \% \text { of patients } \\
\qquad(95 \% \mathrm{Cl})^{\mathrm{b}}\end{array}$ & $\begin{array}{l}\text { Modeled use, \% of patients } \\
\qquad(95 \% \mathrm{Cl})^{c}\end{array}$ \\
\hline$<10 \%$ & 422 & $48.3(35.9-60.8)$ & $49.1(39.5-58.7)$ \\
\hline $20 \%$ to $<30 \%$ & 281 & $36.3(16.0-56.5)$ & $40.4(30.1-50.8)$ \\
\hline
\end{tabular}

aPercentage of bronchiolitis admissions out of unit's total admissions.

b Width of $95 \% \mathrm{Cl}$ accounts for within-hospital clustering.

The mixed effects model used a random intercept for each hospital to account for hospital-level variation. It is adjusted for covariates identified in the primary study's analysis (age combined with preterm birth, time since weaning from supplemental oxygen or flow, documented history of apnea or cyanosis during the present illness, presence of an enteral feeding tube, history of neurologic impairment, and nighttime observation).

included only if they were not receiving supplemental oxygen or nasal cannula flow at the time of data collection. The inclusion and exclusion criteria were for both the parent study and the substudy. Further inclusion and exclusion criteria have been described previously. ${ }^{10,12}$

\section{Data Collection}

In order to ascertain continuous pulse oximetry monitoring status, staff at each hospital performed observational rounds by walking to the bedside of each patient who met inclusion criteria. Additional methodology for the parent study has been published elsewhere..$^{10,12}$

\section{Bronchiolitis Admission Volume by Unit}

Collaborators at each hospital gathered bronchiolitis census data from each unit that admitted patients with bronchiolitis. Units were identified prior to data collection and were characterized at the institution level based on previous local definitions. Each site was responsible for using institution-specific data collection methods for determining bronchiolitis and total admissions on each unit (eg, departmental reports or directly querying admissions data using International Classification of Diseases, Tenth Revision, diagnosis codes for bronchiolitis) over the same period as the parent study. Following data analysis, bronchiolitis admission burden was classified into five categories, based on less than $10 \%, 10 \%$ to less than $20 \%, 20 \%$ to less than $30 \%, 30 \%$ to less than $40 \%$, or $40 \%$ or more of total admissions having a primary discharge diagnosis of bronchiolitis during the study period. This categorization allowed investigators to determine whether there was a dose-dependent response among categories.

\section{Unit Composition}

Site investigators also completed a survey identifying which patients were admitted to each unit (eg, general pediatrics only, medical subspecialty, surgical). Based on these results, units were further classified into seven types (Appendix Table). For the final analysis, units caring exclusively for general pediatrics patients were compared to all other unit types.

\section{Analysis}

Bronchiolitis admission burden and unit composition data were combined with observations of pulse oximetry monitoring use of patients not requiring supplemental oxygen from the parent study. We determined unadjusted observed monitoring proportions for each unit's bronchiolitis admission burden category across all 25 hospitals. This was calculated as a simple proportion of the total number of observations during which patients were continuously monitored divided by the total number of observations performed within each unit's admission category. We then calculated unadjusted odds ratios using the $40 \%$ and higher bronchiolitis admission burden category as a reference. We calculated similar proportions and odds ratios for the dichotomous unit composition variable. Next, we used mixed-effects logistic regression with a random intercept for each hospital to allow for differences in baseline monitoring rates, which varied widely between hospitals ( $2 \%$ to $92 \%),{ }_{10}^{10}$ to calculate adjusted odds ratios for the unit's admission category and unit's composition. We also adjusted for the same covariates used in the primary study's analysis (Table). ${ }^{10}$

\section{RESULTS}

We analyzed 2,366 observations of bronchiolitis patients from 25 hospitals. Most observations were concentrated in freestanding children's hospitals (89\%), and $50 \%$ were from hospitals with more than 250 pediatric beds. Observations were well distributed among the five categories of admit burden (Table).

In unadjusted regression, the relationship between admission burden and rate of pulse oximetry use did not appear to be dose-dependent, and $95 \% \mathrm{Cls}$ were wide. We then analyzed the data accounting for baseline differences in hospital monitoring rates and adjusted for the covariates significantly associated with continuous pulse oximetry monitoring in the primary study's analysis with use of a mixed-effects model. As shown in the Table, low-burden units in which bronchiolitis constituted less than $10 \%$ of total admissions had a 2.16fold increased odds of unnecessary pulse oximetry monitoring compared to high-burden units in which bronchiolitis constituted $40 \%$ or more of total admissions $(95 \% \mathrm{Cl}, 1.27-3.69 ; P=$ 
$.01)$.

In examining the subspecialty unit composition, 596 observations (25.2\%) were conducted on units exclusively caring for general pediatrics patients. In the mixed-effects model adjusted for bronchiolitis admission burden and the covariates used in the study's primary analysis, units exclusively caring for general pediatrics patients did not have significantly different independent odds of pulse oximetry monitoring use compared to units with a mixed patient population (OR $1.01 ; 95 \% \mathrm{Cl}, 0.71$ 1.45; $P=$.95) (Appendix Table).

\section{DISCUSSION}

In this multicenter observational study of children hospitalized with bronchiolitis not concurrently receiving supplemental oxygen, units that only occasionally cared for bronchiolitis patients appeared to be more likely to overuse continuous pulse oximetry during bronchiolitis hospitalizations.

This finding was not immediately apparent when examining the raw data because of wide hospital-level variation in continuous pulse oximetry monitoring use. However, when the high degree of hospital-level variation in baseline overuse was accounted for with use of a random intercept for each hospital in the mixed-effects model, units that cared for higher proportions of bronchiolitis patients had significantly lower odds of continuous pulse oximetry monitoring use compared to units that cared for these infants infrequently.

As many institutions have subspecialized units to cultivate nursing expertise for care of certain diseases and patient populations, we hypothesized that units caring primarily for children on general pediatrics units would also have lower rates of monitoring overuse compared to mixed units. Interestingly, these units did not perform better, likely because potential cultural factors that might contribute to differences in monitoring are accounted for by bronchiolitis admission burden.

Our findings build on prior literature by demonstrating that unit-level, as well as hospital-level, factors appear to drive overuse in healthcare. A prior single-site retrospective cohort study demonstrated an association between higher prevalence of inpatient bronchiolitis and decreased use of unnecessary interventions such as laboratory and radiographic testing, as well as steroid and antibiotic administration. ${ }^{11}$ Although study of the relationship between volume and quality is not new to healthcare, to our knowledge, this study is the first to examine the relationship between pulse oximetry overuse in bronchiolitis and unit-level factors like admission burden and subspecialty composition.

There are several limitations. First, because the study population included only children not receiving supplemental oxygen, both the parent study and this substudy assumed that all observed use of pulse oximetry monitoring was overuse. In some cases, however, there may have been other compelling clinical reasons, institutional policies, or differences in pulse oximetry availability that were not captured during data collection or in our adjusted model. Second, hospitals used convenience sampling. It is possible this resulted in samples that were not representative of each unit's underlying patient pop- ulation or monitoring practice. In addition, not all of the 32 eligible sites were able to provide data related to hospital admissions at the unit level and thus are not included in our analysis. This remains a potential source of hospital-level selection bias.

\section{CONCLUSION}

These findings demonstrate that high bronchiolitis admission burden correlates with lower rates of unnecessary pulse oximetry monitoring in bronchiolitis. We speculate that these outcomes might reflect differing degrees of nursing comfort, expertise, and unit-level norms in caring for bronchiolitis patients, although our study was not designed to establish underlying causes. Identification of operating principles that underpin low pulse oximetry monitoring on high-burden units will provide guidance for decreasing unnecessary monitoring and will inform future studies seeking ways to discourage continuous pulse oximetry monitoring in low-risk infants. Given the institutional variation in monitoring rates, future studies examining both institution-wide and unit-level interventions will be necessary to decrease unnecessary pulse oximetry monitoring in bronchiolitis. Furthermore, these findings may be relevant to studying care quality in other disease processes, with bronchiolitis serving as a model illness for overuse.

\section{Acknowledgments}

The authors acknowledge the National Heart, Lung, and Blood Institute of the National Institutes of Health scientists who contributed their expertise to this project as part of the U01 Cooperative Agreement funding mechanism as federal employees conducting their official job duties: Lora Reineck, MD, MS, Karen Bienstock, MS, and Cheryl Boyce, PhD. The authors thank the executive council of the Pediatric Research in Inpatient Settings Network for their contributions to the early scientific development of this project. The network assessed a Collaborative Support Fee for access to the hospitals and support of this project.

The authors thank the PRIS Network collaborators for their major contributions to data collection (see Appendix).

Disclosures: The authors have no conflicts of interest relevant to this article to disclose.

Funding: Research reported in this publication was supported by a Cooperative Agreement from the National Heart, Lung, and Blood Institute of the National Institutes of Health under award number U01HL143475 (Dr Bonafide, Principal investigator). The funding organization had no role in the design of the study; collection, management, analysis, and interpretation of the data; preparation, review, or approval of the manuscript; or decision to submit the manuscript for publication. The content is solely the responsibility of the authors and does not necessarily represent the official views of the National Institutes of Health.

\section{References}

1. Ralston SL, Lieberthal AS, Meissner HC, et al. Clinical practice guideline: the diagnosis, management, and prevention of bronchiolitis. Pediatrics. 2014;134(5):e1474-e1502. https://doi.org/10.1542/peds.2014-2742

2. Quinonez RA, Garber MD, Schroeder AR, et al. Choosing wisely in pediatric hospital medicine: Five opportunities for improved healthcare value. J Hosp Med. 2013;8(9):479-485. https://doi.org/10.1002/jhm.2064

3. Quinonez RA, Coon ER, Schroeder AR, Moyer VA. When technology creates uncertainty: pulse oximetry and overdiagnosis of hypoxaemia in bronchiolitis. BMJ. 2017;358:j3850. https://doi.org/10.1136/bmj.j3850

4. Cunningham S, Rodriguez A, Adams T, et al; Bronchiolitis of Infancy Discharge Study (BIDS) group. Oxygen saturation targets in infants with bronchiolitis (BIDS): a double-blind, randomised, equivalence trial. Lancet. 
2015;386(9998):1041-1048. https://doi.org/10.1016/s0140-6736(15)00163-4

5. Schroeder AR, Marmor AK, Pantell RH, Newman TB. Impact of pulse oximetry and oxygen therapy on length of stay in bronchiolitis hospitalizations. Arch Pediatr Adolesc Med. 2004;158(6):527-530. https://doi.org/10.1001/ archpedi.158.6.527

6. Cunningham S, McMurray A. Observational study of two oxygen saturation targets for discharge in bronchiolitis. Arch Dis Child. 2012;97(4):361-363. https://doi.org/10.1136/adc.2010.205211

7. Cunningham S, Rodriguez A, Boyd KA, Mclntosh E, Lewis SC; BIDS Collaborators Group. Bronchiolitis of Infancy Discharge Study (BIDS): a multicentre, parallel-group, double-blind, randomised controlled, equivalence trial with economic evaluation. Health Technol Assess. 2015;19(71):i-172. https://doi. org/10.3310/hta19710

8. Bonafide CP, Lin R, Zander M, et al. Association between exposure to nonactionable physiologic monitor alarms and response time in a children's hospital. J Hosp Med. 2015;10(6):345-351. https://doi.org/10.1002/jhm.2331
9. Ralston SL, Garber MD, Rice-Conboy E, et al. A multicenter collaborative to reduce unnecessary care in inpatient bronchiolitis. Pediatrics. 2016;137(1):e20150851. https://doi.org/10.1542/peds.2015-0851

10. Bonafide CP, Xiao R, Brady PW, et al; for the Pediatric Research in Inpatient Settings (PRIS) Network. Prevalence of continuous pulse oximetry monitoring in hospitalized children with bronchiolitis not requiring supplemental oxygen. JAMA. 2020;323(15):1467-1477. https://doi.org/10.1001/jama.2020.2998

11. Van Cleve WC, Christakis DA. Unnecessary care for bronchiolitis decreases with increasing inpatient prevalence of bronchiolitis. Pediatrics. 2011;128(5):e1106-e1112. https://doi.org/10.1542/peds.2011-0655

12. Rasooly IR, Beidas RS, Wolk CB, et al. Measuring overuse of continuous pulse oximetry in bronchiolitis and developing strategies for large-scale deimplementation: study protocol for a feasibility trial. Pilot Feasibility Stud. 2019;5(1):68. https://doi.org/10.1186/s40814-019-0453-2 